\title{
Prehospital Indicators for Disaster Preparedness and Response: New York City Emergency Medical Services in Hurricane Sandy
}

Silas W. Smith, MD; James Braun, MA; Ian Portelli, PhD, MSc; Sidrah Malik, MPH, MHA; Glenn Asaeda, MD; Elizabeth Lancet, MPH; Binhuan Wang, PhD; Ming Hu, PhD;

David C. Lee, MD, MS; David J. Prezant, MD; Lewis R. Goldfrank, MD

\section{ABSTRACT}

Objective: We aimed to evaluate emergency medical services (EMS) data as disaster metrics and to assess stress in surrounding hospitals and a municipal network after the closure of Bellevue Hospital during Hurricane Sandy in 2012.

Methods: We retrospectively reviewed EMS activity and call types within New York City's 911 computerassisted dispatch database from January 1, 2011, to December 31, 2013. We evaluated EMS ambulance transports to individual hospitals during Bellevue's closure and incremental recovery from urgent care capacity, to freestanding emergency department (ED) capability, freestanding ED with 911-receiving designation, and return of inpatient services.

Results: A total of 2,877,087 patient transports were available for analysis; a total of 707,593 involved Manhattan hospitals. The 911 ambulance transports disproportionately increased at the 3 closest hospitals by $63.6 \%, 60.7 \%$, and $37.2 \%$. When Bellevue closed, transports to specific hospitals increased by $45 \%$ or more for the following call types: blunt traumatic injury, drugs and alcohol, cardiac conditions, difficulty breathing, "pedestrian struck," unconsciousness, altered mental status, and emotionally disturbed persons.

Conclusions: EMS data identified hospitals with disproportionately increased patient loads after Hurricane Sandy. Loss of Bellevue, a public, safety net medical center, produced statistically significant increases in specific types of medical and trauma transports at surrounding hospitals. Focused redeployment of human, economic, and social capital across hospital systems may be required to expedite regional health care systems recovery. (Disaster Med Public Health Preparedness. 2016;10:333-343)

Key Words: disaster, emergency medical services, emergency departments, Hurricane Sandy, prehospital, warning

$\mathrm{T}$ he Institute of Medicine has determined that the ongoing loss of emergency care and trauma capacity and the resultant overcrowding have left both prehospital and hospital services stressed and vulnerable to natural and human-engineered disasters. ${ }^{1,2}$ Climate change and geopolitical turmoil suggest that the impact of disaster is likely to increase. The emergency services and health care and public health sectors are recognized as critical infrastructure and key resources by the National Infrastructure Protection Plan. ${ }^{3}$ As the diversity of public health emergencies continues to challenge societies, most recently evidenced by the deadliest Hajj disaster in history, a thorough understanding of the capacity and adaptability of emergency services and health care systems is critical for disaster preparedness and mitigation. ${ }^{4,5}$ However, despite Hospital Preparedness Program resource allocations, definitive metrics to assess the preparedness of health systems remain provisional. ${ }^{6}$

Hurricane Sandy, the second costliest hurricane in US history, made landfall on October 29, 2012. Sandy decimated 3 lower Manhattan hospitals, including a regional trauma center, a comprehensive psychiatric emergency program (CPEP), and a "safety net" academic medical center at Bellevue Hospital Center; the ST-segment elevation myocardial infarction (STEMI) and stroke receiving centers at NYU Langone Medical Center Tisch Hospital (NYULMCTH) and Bellevue; and the Manhattan Campus of the Veterans' Administration Medical Center (see the map in online data supplement 1). Physically intact, proximate hospitals were stressed not only by the requirement to absorb these hospitals' more than 750 collective evacuees but also by the need to substitute 
care for medical campuses providing for greater than 800,000 outpatient visits and more than 150,000 emergency department (ED) visits per year. Bellevue regained inpatient functions on February 7, 2013; NYULMCTH remained without an ED until April 22, 2014; and the Veterans' Administration Medical Center was without inpatient services until May 21, 2013. New York-Presbyterian/Lower Manhattan Hospital (NYP/LMH), a 180-bed community hospital with approximately 32,000 ED visits per year, evacuated and closed for 5 days. In addition to Bellevue, the New York City Health and Hospitals Corporation (HHC), the country's largest municipal health system, suffered the loss of Coney Island Hospital in Brooklyn, an outer borough of New York City, which did not reopen until June 11, 2013.

In a stakeholders meeting convened by the Institute of Medicine and the New York Academy of Medicine at the request of the US Department of Health and Human Services (DHHS) barely 2 weeks after Hurricane Sandy, priority research questions and information gaps already included cross-sector essential information and data sharing, available databases and information sources, and effective deployment strategies for health workforce personnel. ${ }^{5,7}$ Emergency medical services (EMS) data may provide a previously underappreciated resource for objective measures for disaster preparedness and response. ${ }^{6}$ These data may provide rapidly accessible indicators and triggers to deploy resources or to implement crisis standards of care. ${ }^{1}$ We previously identified the utility of hospital-based ED metrics as clear evidence of the need to restore emergency services at Bellevue by means a novel freestanding, 911-receiving ED prior to hospital reopening. 8 Analysis of prehospital 911 call volumes, including analyses of specific call subtypes and the distribution of high-acuity groups, high-priority calls, and behavioral health calls could be particularly relevant for assessing storm impact and potential early markers of systemic stress.

Given that historical hospital catchment zones are well defined, alterations in transports to specific hospitals in the aftermath of a natural disaster might serve as a proxy indicator of systemic decompensation. Examination of adaptive patterns might also illustrate key effects across a health care system from actions occurring at specific elements within it. We explored the response of a complex regional health system at the prehospital level and assessed pre- and post-disaster functional capacity. These data have the potential to inform preparedness for and response to future large-scale disasters.

\section{METHODS}

\section{Study Setting and Population}

This was a retrospective review of EMS activity within New York City's 911 emergency dispatch system. The Fire Department of New York City (FDNY) is the primary provider of prehospital emergency care in NYC's 5 boroughs and is the country's largest fire department-based EMS and the largest combined fire and EMS provider in the world. FDNY serves an area greater than 300 square miles and a population of over 8 million people, a number that increases during business hours to over 9 million. NYC's EMS has utilization rates nearly twice those of the rest of the nation. ${ }^{9}$ FDNY's Division of EMS has more than 2400 emergency medical technicians staffing basic life support and over 870 paramedics staffing advanced life support. In 2012, the FDNY-EMS 911 emergency dispatch system handled over 1.3 million EMS calls, which resulted in more than 900,000 patient transports per year. Not all calls result in transports to hospitals because patients may refuse medical aid or transportation, callers may not be present upon EMS arrival, or calls may be unfounded.

\section{Data Source}

FDNY-EMS maintains a robust, computer-assisted dispatch database that contains over 15 years of data. ${ }^{9-11}$ The database has demonstrated the capability to compare short- and longterm temporal trends and has been previously utilized to evaluate urban crisis events, such as citywide blackouts. ${ }^{9-11}$

Emergency medical dispatchers use a prioritization algorithm to classify incoming 911 EMS calls into 1 of 74 distinct primary dispatch call types, several with subdesignations (see online data supplement 2), that reflect the nature of the medical or trauma emergency. The algorithm has remained consistent for 15 years, with the exception of the creation of a mass casualty incident call type in 2000, the addition of fevercough and fever-rash designations in 2002 to address the threat of biological terrorist attacks after 9/11, an asthma reclassification in 2007, and the addition of fever-travel designations in the wake of Ebola. To provide a meaningful framework and correlate with the spectrum of diseases, call types are classified into 6 broad tertiary medical categories and 4 broad tertiary trauma categories and then into secondary and primary medical and trauma groupings. Call types are assigned a priority ("segment"), which approximates acuity. Call distribution and subtype analysis can be evaluated, specifically to determine distribution alterations in the face of unanticipated disaster. ${ }^{10,11}$ These classification subtypes have also been shown to be representative of ED visits for similar EMS complaints, providing a suitable substitute to evaluate system-wide effects. ${ }^{9}$ Ambulance crews can update the dispatch-assigned call type to a "final call type" to reflect their clinical assessments and interventions.

EMS transports to specific hospitals were evaluated as indicators of prehospital utilization and systemic decompensation. These transports were analyzed in 4 different periods defined by care capacity at Bellevue: (1) closure upon Sandy landfall to urgent care capacity on November 19, 2012; (2) freestanding ED capability on December 10, 2012; (3) freestanding ED with 911-receiving designation on December 24, 2012; and (4) return of full services including inpatient care on 
February 7, 2013. The periods were compared to the same time periods 1 year prior. Specialty hospitals (eg, surgical subspecialty, oncological, and Veterans Administration) not classified as general, 911-receiving hospitals were excluded from analysis.

\section{Data Analysis}

We analyzed post-storm prehospital transport calls, hospital distributions, and call subtypes and compared them to historical trends, with specific attention to hospitals in Manhattan and the New York City HHC network, the country's largest municipal health system, where Bellevue staff were redeployed. For call type analyses, we utilized "final call type" as described above. In a de-identified, aggregate format, a descriptive statistical analysis of these data was performed. Previous studies have demonstrated that analyses of individual call types in the 911 dataset are amenable to using a $t$-test with presumed equal variance. ${ }^{10}$ We re-verified that the variances of the groups were equal by using Levene's test. We subsequently analyzed the prehospital transports to individual hospitals by applying a two-sided, independent samples $t$-test. These data were analyzed by using SPSS version 21.0.0.0 (IBM Corp, Armonk, NY). SAS software version 9.3 (SAS Institute Inc, Cary, NC) was used to analyze individual final primary call types-hospital pairs; a univariant linear regression model was used to fit pre/ post Sandy call data. We used pre-Sandy call data to predict post-Sandy call data, and reported the $95 \%$ prediction confidence intervals. Outlier and leverage diagnostics were used to aid identification of call types with statistically significant differences or effects. A two-sided paired $t$-test was used to evaluate statistical significance.

\section{Ethics}

The conduct of this study was approved by the Institutional Review Boards of the NYU School of Medicine, Office of Science and Research, and the New York City Health and Hospitals Corporation.

\section{RESULTS}

For the period of January 1, 2011, through December 31, 2013, a total of 2,877,087 city-wide records (patient transports) were available for analysis to evaluate the impact of Bellevue's closure on HHC hospitals. Of these transports, 707,593 were restricted to Manhattan hospitals.

Bellevue's closure after Hurricane Sandy's landfall produced a rapid and sustained rise in prehospital EMS transports to the closest alternative Manhattan hospital destinations (Figure 1). Transports to proximate hospitals returned to baseline after Bellevue's reopening. This was despite NYULMCTH remaining closed to 911 ambulances throughout this study period and not reopening until 2014. As is evident in the temporal trends shown in Figure 1, although NYP/LMH (2.80 miles distant from Bellevue) evacuated and closed for 5 days, it was a relatively small community hospital and therefore its reopening did not significantly impact nearby prehospital transports.

We focused on mean ambulance transports for the time period from Bellevue hospital closure until February 7, 2013, when Bellevue resumed full inpatient operations, and compared these to the same period 1 year prior. Overall, mean 911 ambulance transports per day increased at the closest hospital, Mount Sinai Beth Israel (MSBI, 0.66 miles distant) by 63.6\%, from 76.1 to $124.6(P<0.001)$; at New York-Presbyterian/ Weill Cornell Medical Center (NYP/WCMC, 2.26 miles distant) by $60.7 \%$, from 44.2 to $71.1 \quad(P<0.001)$; and at Mount Sinai Roosevelt (MSR, 4.7 miles distant) by 37.2\%, from 56.8 to $78.0(P<0.001)$. For the first 2 months, Bellevue could not participate in the 911 system. When its freestanding ED was approved as a 911-receiving ED, it was restricted from receiving trauma, cardiac arrest, third-trimester pregnancy, STEMI, stroke, obvious surgical disease, emotional disturbance, and those in police custody until full inpatient services were restored. Despite these considerable restrictions, with freestanding, 911-receiving designation, Bellevue received mean transports of $37 \%$ of baseline ( 29.3 compared to 78.5, $P<0.001$ ), which did blunt the increase in mean EMS transports to MSBI, the nearest hospital, to 110.8 per day $(P<0.001$ by two-sided, independent samples $t$-test $)$.

The database was sufficiently robust to evaluate even minimal changes from baseline. For example, there was a statistically significant but clinically insignificant rise of 3.8 ambulances per day from 36.3 to 40.1 ambulances $(P=0.006)$ at NYP/ $\mathrm{LMH}$. There was a statistically significant but clinically insignificant increase of 3.4 ambulances per day from 22.4 to 25.8 ambulances per day $(P<0.001)$ at Lennox Hill Hospital, and a rise of 3.5 ambulances per day 44.1 to 47.6 ambulances per day $(P=0.001)$ at Mt. Sinai Hospital. Although 911 ambulance transports did increase at New York-Presbyterian/Columbia University Medical Center (NYP/CUMC) from 63.0 to 71.1 ambulances per day $(P<0.001)$, this difference primarily occurred after December 10, 2013, more than 40 days after Hurricane Sandy landfall. Other northern Manhattan hospitals saw no clinically or statistically significant rises in ambulance transports: from 31.83 to $33.03(P=0.171)$ at Metropolitan Hospital Center, from 46.04 to $46.58(P=0.602)$ at Harlem Hospital Center, from 67.58 to $68.31(P=0.600)$ at Mount Sinai St. Luke's, and from 25.83 to $26.53(P=0.377)$ at New York-Presbyterian/The Allen Hospital.

In addition to Bellevue, the municipal HHC network also suffered the loss of Coney Island Hospital. Figure 2 depicts the effect on prehospital transports to HHC hospitals following Hurricane Sandy, which overall decreased by $13.9 \%$ from the year prior during the period of October 29, 2012, to February 6, 2013, from 738.2 to 635.3 per day $(P<0.001)$. Transports to hospitals closest to Coney Island HospitalKings County Hospital Center (5.1 miles), Woodhull 
FIGURE 1

FDNY EMS Transports to Manhattan Receiving Hospitals January 2011 Through December 2013.

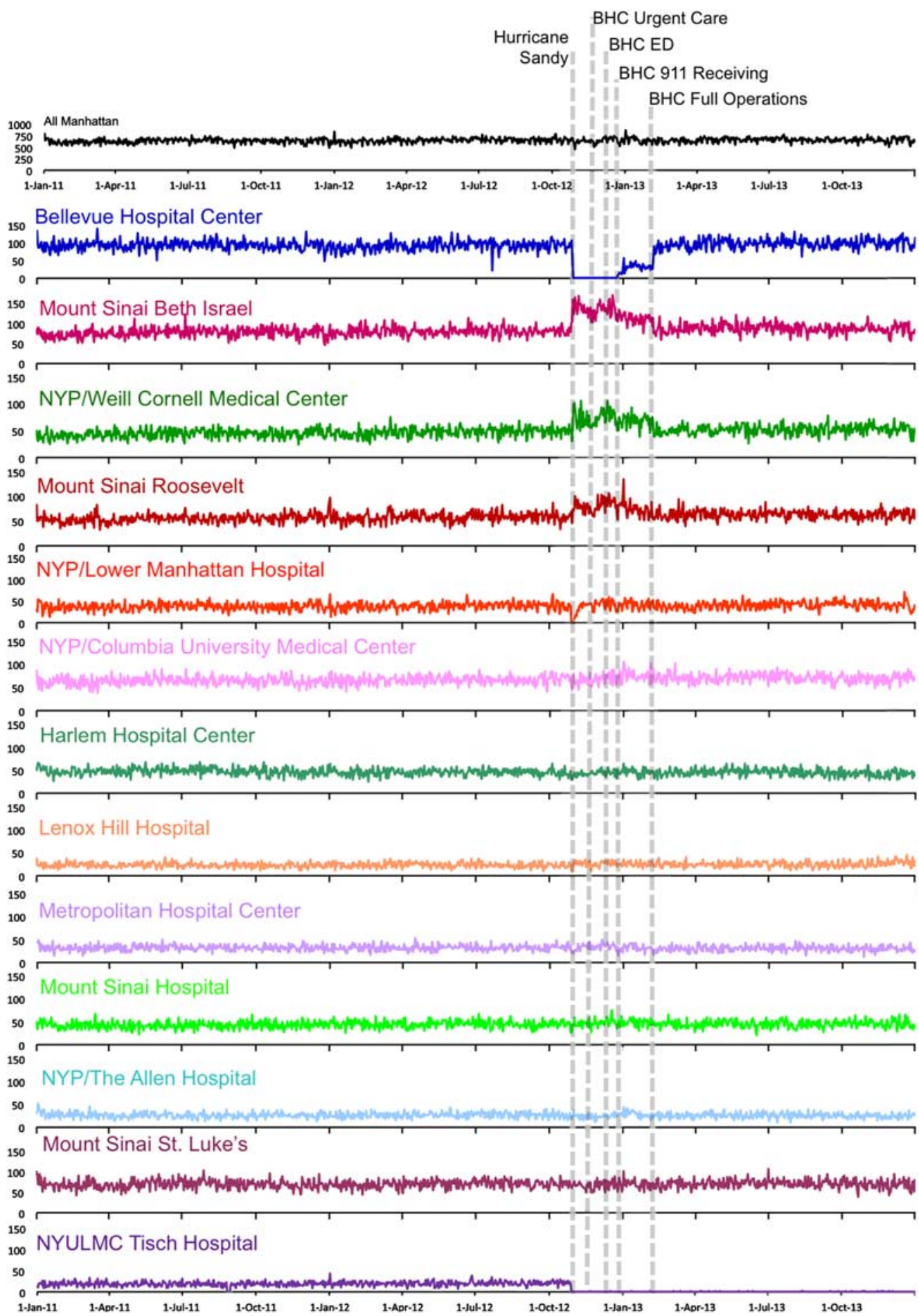

The negligible transports to NYULMCTH during August 26-29, 2011, reflects evacuation and shelter-in-place for Hurricane Irene. Beth Israel Medical Center became Mount Sinai Beth Israel following the Continuum Health Partners-Mount Sinai Medical Center merger in September 2013; Mount Sinai Roosevelt assumed its name January 2014. New York Downtown Hospital became New York Presbyterian (NYP)/Lower Manhattan Hospital after its 2013 merger with NYP. Abbreviations: BHC, Bellevue Hospital Center; ED, emergency department; EMS, emergency medical services; FDNY, Fire Department of New York City; NYULMC, NYU Langone Medical Center. 
FGURE 2

FDNY EMS Transports to New York City Health and Hospitals Corporation Hospitals January 2011 Through December 2013.

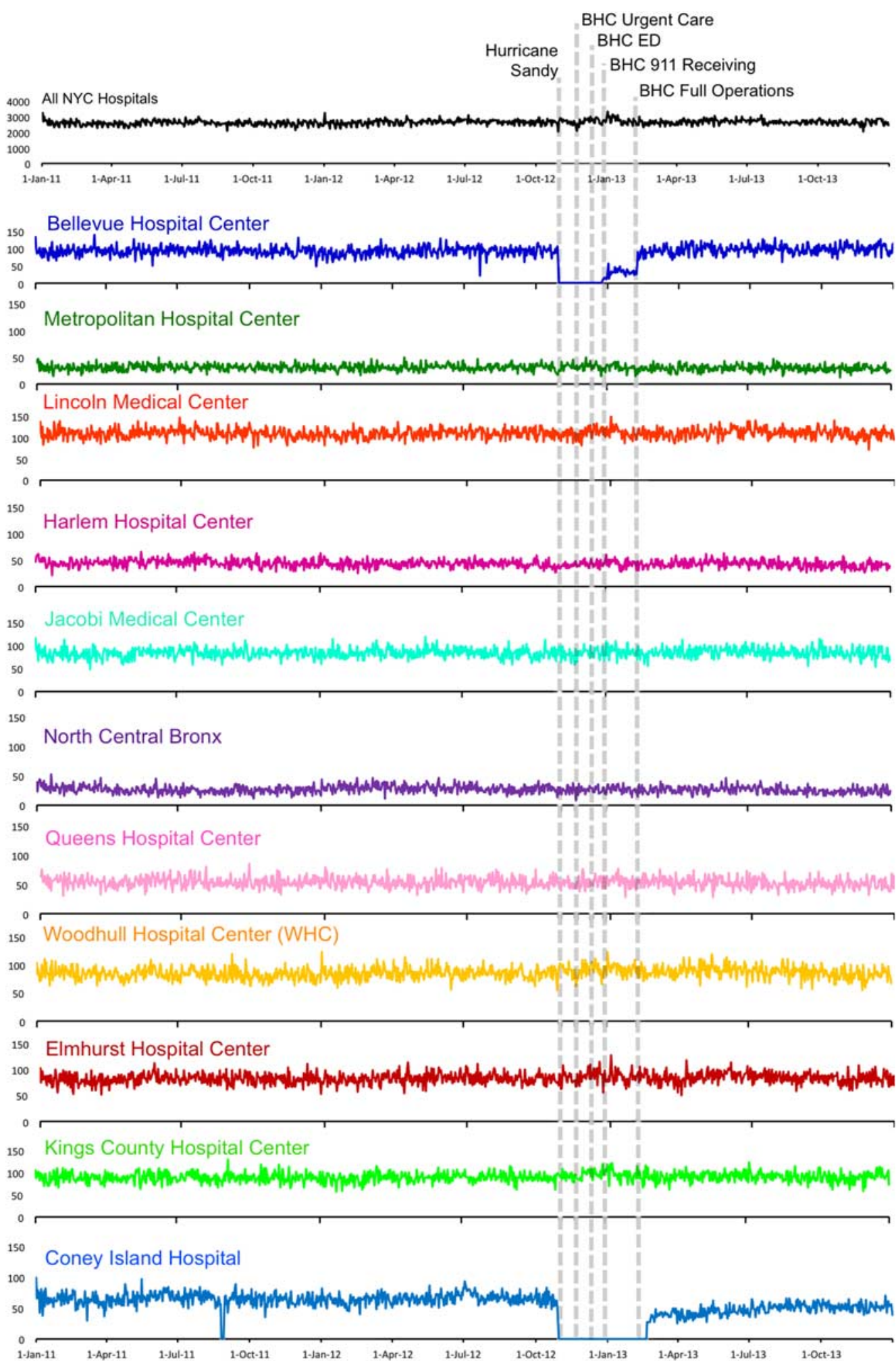

The absence of transports to Coney Island Hospital from August 26-28, 2011, reflects a planned evacuation due to Hurricane Irene. Abbreviations: BHC, Bellevue Hospital Center; ED, emergency department; EMS, emergency medical services; FDNY, Fire Department of New York City. 
Hospital Center (8.1 miles), and Elmhurst Hospital Center (12.2 miles) - increased by $8.5 \%$ (91.94 to $99.71, P<0.001$ ), $10.3 \%$ (86.19 to $95.06, P<0.001$ ), and $7.6 \%$ (85.55 to 92.04 , $P<0.001$ ), respectively, but this did not compensate for the overall loss of transports to Bellevue and Coney Island. Lincoln Medical Center in the Bronx saw a statistically significant but clinically insubstantial rise of 5.9 ambulances per day from 111.8 to $117.7(P<0.001)$. Harlem Hospital Center, Jacobi Medical Center, Metropolitan Hospital Center (the closest HHC Manhattan hospital to Bellevue), North Central Bronx Hospital, and Queens Hospital Center did not see clinically or statistically significant increases in ambulance transports.

Statistically significant changes occurred in hospital medical and trauma call subtypes. With Bellevue closed and the loss of its STEMI, CPEP, and safety net capacity, there were substantial statistically significant increases (ie, exceeding 95\% prediction limits) in specific medical call type transports to neighboring hospitals: altered mental status (increase of $115.6 \%$ at MSBI), drug and alcohol conditions (increases of $45.7 \%$ at MSBI and $60.0 \%$ at MSR), cardiac conditions (increase of $92.1 \%$ at MSBI), difficulty breathing (increases of $75.2 \%$ at MSBI and $62.0 \%$ at MSR), emotionally disturbed persons (increases of $47.3 \%$ at MSBI and $72.0 \%$ at Mt. Sinai), generalized illness (increases of $62.0 \%$ at MSBI and $74.2 \%$ at NYP/WCMC), and unconsciousness (increases of $55.4 \%$ at MSBI and $100.0 \%$ at NYP/WCMC) (Figure $3 \mathrm{~A}$ ). The clinically and statistically significant trends for these categories persisted until full medical, STEMI, and CPEP services were reinstated when Bellevue reopened its inpatient services (Figures 3B-D).

When Bellevue was closed, the loss of its regional trauma center capacity immediately and statistically significantly increased trauma call type transports for "pedestrian struck" and blunt, nonvehicular traumatic injury at the 2 closest sites, MSBI (increase of $82.7 \%$ for nonvehicular traumatic injury) and NYP/WCMC (increases of $136.4 \%$ for pedestrian struck and $54.2 \%$ nonvehicular traumatic injury), despite MSBI's lack of a trauma center designation (Figure 4A). Trauma call transports actually significantly decreased at hospitals further afield, specifically, blunt, nonvehicular traumatic injury at Harlem Hospital Center (decrease of 15.1\%), Mount Sinai St. Luke's (decrease of $16.7 \%$ ), NYP/LMH (decrease of $18.7 \%$ ), and NYP/CUMC (decrease of 25.6\%). The clinically and statistically significant trends persisted until full trauma center capabilities were reinstated when Bellevue reopened its inpatient services (Figures 4B-D). Overall, during the time periods before Bellevue's full restoration of inpatient and trauma services (Figures 4A-D), with Bellevue receiving no ambulance trauma, MSBI and NYP/WCMC blunt trauma nonvehicular injury transports increased from 918 to 1571 (71.1\%) and from 853 to 1359 (59.3\%), respectively. At NYP/WCMC, "pedestrian struck" calls increased 107.5\% from 159 to 330.

\section{DISCUSSION}

FDNY EMS data provided robust, identifiable, concrete markers of Hurricane Sandy's impact on health care systems. EMS data obviated the overwhelmingly complex demand of acquiring and synthesizing ambulance arrival data from over 65 New York City hospitals in the 911 system. Our methods advance prior recommendations that municipal operational databases should be developed and maintained as valuable all-hazards resources for real-time decision-making and disaster analysis. ${ }^{5}$ At the federal level, our work implies that data from the emergency services sector (with Department of Homeland Security as its sector-specific agency) can inform response in the health care and public health sector (with DHHS as the sector-specific agency); inter-agency data sharing should be facilitated.

Prior studies have demonstrated global increases in EMS volumes during periods of prolonged environmental stress (eg, urban heat waves). ${ }^{12}$ In a controlled ED closure and consolidation at Boston Medical Center, EMS data demonstrated an increased mean turnaround interval in 5338 EMS transports after closure. ${ }^{13}$ However, that study was unable to assess the full systemic impact at other hospitals owing to an inability to evaluate their corresponding $\mathrm{ED}$ volumes. We extended the evaluation of volumes to specific hospitals during a prolonged disaster. In the current study, loss of a major public safety net hospital created systemic stress across other 911-receiving institutions. The closest hospitals bore a disproportionate increase in prehospital transports during Hurricane Sandy and its prolonged aftermath. These hospitals were distinct from hospitals to which closed hospitals - both private (NYULMCTH) and public (Bellevue) - proportionally redeployed their emergency staff. These results expand the observations associated with short-term events and predicted through modeling: that patients are transported to the closest or familiar hospitals in disaster. ${ }^{14,15}$ Additionally, these results are comparable to those reported when Saint Vincent's Hospital, a regional trauma center in lower Manhattan, abruptly ceased operations and permanently closed in April 2010. Sudden and sustained surges in patient volume and critical care occurred in 4 surrounding hospitals, particularly in the sole remaining regional trauma center, and patients experienced reduced access to care and prolonged travel times. ${ }^{16,17}$

Hurricane Sandy confirmed the Institute of Medicine's conclusion that years of deteriorating capacity for emergency and trauma services have left the remaining hospital services system vulnerable to becoming overwhelmed by natural disasters. ${ }^{1,2}$ As has been suggested for acute disaster events, ${ }^{14}$ our findings emphasize the need to develop flexible prehospital routing strategies for ambulances with less critical patients to more distant hospital destinations or catchment zone shifting to lessen surge in EDs already receiving higher walk-in volumes. In addition, to ensure that referral hospitals with unique service capacities such as advanced trauma care are not overwhelmed, a dynamic prehospital response may be 
FDNY EMS Primary Medical Call Types.
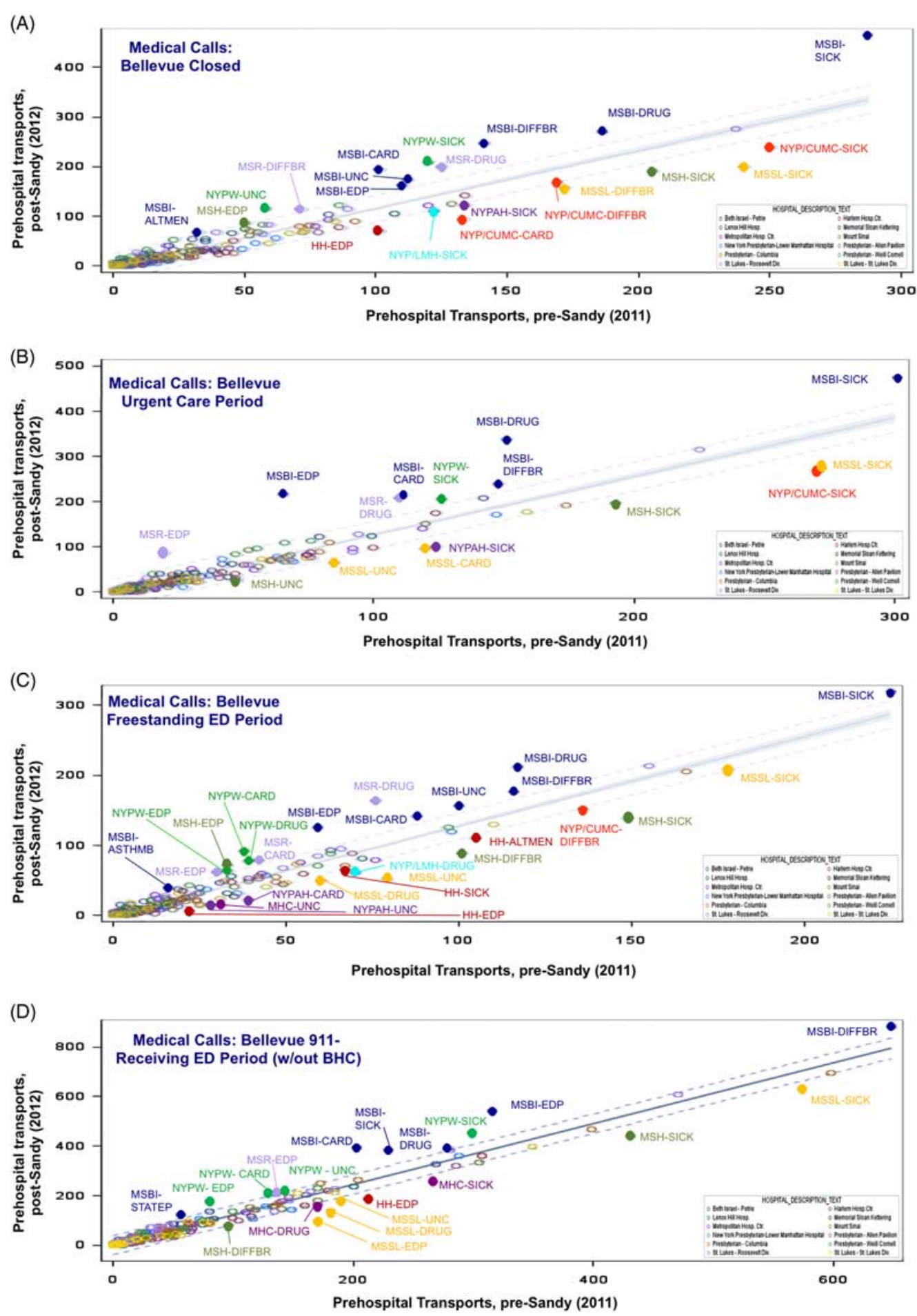

Each point represents a specific call type transported to a specific destination hospital during Bellevue (A) closure, (B) urgent care status, (C) freestanding ED status, and (D) 911-receiving ED status. The ordinate is the number of transports for the specified post-Sandy period; the abscissa is transports for the identical time period in the prior year. Dotted lines represent $95 \%$ prediction limits. Selected points are highlighted, which represent statistically significant differences in transports. Abbreviations: ALTMEN, altered mental status; ASTHMB, asthma/BLS; BHC, Bellevue Hospital Center; CARD, cardiac condition; DIFFBR, difficulty breathing; DRUG, drug or alcohol abuse; ED, emergency department; EDP, emotionally disturbed person; EMS, emergency medical services; FDNY, Fire Department of New York City; HH, Harlem Hospital Center; SICK, generalized illness; UNC, unconscious. 
FIGURE 4

Primary Trauma Call Types.


Each point represents a specific call type transported to a specific destination hospital during Bellevue (A) closure, (B) urgent care status,

(C) freestanding ED status, and (D) 911-receiving ED status. The ordinate is the number of transports for the specified post-Sandy period; the abscissa is transports in the prior year. Dotted lines represent $95 \%$ prediction limits. Selected points are highlighted, which represent statistically significant differences in transports. Abbreviations: ED, emergency department; HH, Harlem Hospital Center; INJURY, blunt trauma nonvehicular injury; MVAINJ, motor vehicle accident with injury; OTHER, other traumatic injury; PEDSTR, pedestrian struck. 
required to preserve the efficiency of these vital functions. In other words, the best systems response to hospital closures may not be transport to the next closest hospital if that hospital is already functioning at many-fold its usual capacity.

In addition to volume increases, our analysis identified call types that may strain prehospital resources (Figures 3 and 4). It is important to note that regardless of ultimate outcome or diagnosis, many of these were a priori high-priority calls ("segment" 2 [difficulty breathing, unconsciousness] or "segment" 3 [altered mental status, cardiac conditions, pedestrian struck]) requiring particularly rapid ambulance responses and potential deployment of critical advanced life support resources. Such calls may additionally challenge receiving hospitals owing to their resource-intense or time-sensitive nature and the need for coordinated hand-offs from the prehospital to hospital setting. For example, "difficulty breathing" calls may require advanced noninvasive or invasive ventilation and pharmacological interventions. Trauma transports often involve multi-disciplinary hospital systems activations that can extend past the operating room. "Cardiac" conditions may require similar activations, resource-intensive procedural, electrophysiological, or critical care interventions. Unconscious patients or those with altered mental status require swift attention to etiology and treatment. Emotionally disturbed persons require team-based interventions, not infrequently involving law enforcement, to address potentially life-threatening medical or psychiatric conditions and the additional risks posed to providers, other patients, and staff. The increased drug, alcohol, and emotionally disturbed person transports highlight the need to consider these particular medically vulnerable populations, the complex social planning that may be required to ensure safe ED discharge, ${ }^{18-20}$ and the need to maintain robust psychiatric services, resourced for patient care.

Although essential to the individual patient, the very act of prioritization (triage) has the potential to detract from the quality and safety of other ED patients either through resource compromise, adverse events, length of stay, or mortality. ${ }^{21-24}$ These data highlight the fact that simple volume alterations may insufficiently describe hospital ED stress. Increased numbers and increased criticality may reflect a synergistic burden that could create a downward spiral leading to adverse patient care outcomes and hospital staff burnout.

We previously demonstrated that reestablishing pre-storm level care at Bellevue normalized ED volumes at nearby hospitals. ${ }^{8}$ In the present study, we observed the significant effect that a major safety net, academic medical center can have on surrounding prehospital transports, particularly in light of the fact that rapidly reopening NYP/LMH did not decrease the prehospital burden on the other hospitals surrounding Bellevue. Municipalities and disaster planners should carefully consider the potential for "disproportionate impact" from loss of certain hospital assets or capabilities, associated with the importance of the demographics, socioeconomic status, and disease burdens of the hospital population and function. The failure to salvage New Orleans' Charity Hospital in Hurricane Katrina's wake devastated access to care for medically vulnerable populations and saddled surrounding facilities with substantial burdens in uncompensated care. ${ }^{25,26}$ As has been previously suggested, this implies that the health care delivery system and the public health infrastructure must be considered as an integrated whole in planning for, responding to, and recovering from large-scale disasters. ${ }^{27}$ Hospitals that are "engines of health care delivery" should be brought "back on line" expeditiously and receive mitigation and recovery prioritization. Health care consolidation and hospital realignments should not create medical centers "too big to fail" in a disaster, absent explicit contingency strategies.

Until the degradation of afflicted hospitals can be resolved, the public health interest may be best served through a calibrated redeployment of human, economic, and social capital from closed hospitals to EDs or hospitals with demonstrable increases in volume or criticality. This top-level health care systems resource-sharing requirement necessary for rapid regional recovery may conflict with existing administrative, regulatory, credentialing, and legal constraints; intermediate-level interinstitutional agreements and competitive entities; lower-level corporate divisions, collective bargaining agreements, and individual hospital goals; and individual-level concerns such as workplace location and financial burdens. Future disaster planning, policy, indemnification, and funding should incentivize resource sharing across disparate systems at all levels. Mechanisms to improve provider reallocation (through facilitated credentialing of medical and health care staff), ancillary staff support, redeployment of durable medical assets, access to emergency capital, and other mechanisms should be explored. Governmental leadership at local, state, and federal levels may be essential to mitigate some of the aforementioned socioeconomic and political barriers to respond effectively to critical complex disasters.

\section{Limitations}

Because disaster-affected patients may have lacked the ability to access 911 services owing to loss of electrical or telephone services, not all emergent conditions normally transported by EMS may have been captured. Patients may also have taken alternative means of transportation to reach EDs or hospitals known not to receive 911 ambulances (ie, Bellevue from November 19 to December 24, 2012). If anything, this would underestimate the burden experienced in surrounding hospitals, because our previous evaluations demonstrated that ED walk-in volumes were also significant. ${ }^{8}$ Computer-assisted dispatch call types may not match ultimate discharge diagnoses. We attempted to mitigate this by using the final call type as ascertained by the treating ambulance crew. However, many call types are patently obvious (eg, difficulty breathing, 
pedestrian struck, unconsciousness, emotionally disturbed person) and required potentially rapid interventions. Even in the $\mathrm{ED}$, discharge diagnoses are inadequate to permit accurate identification of nonemergency ED visits owing to the limited concordance between presenting complaints and final diagnoses. ${ }^{28}$ This weakness limits the benefit of the discharge diagnosis in determining the full burden of care for clinical assessment, diagnostics, and response to therapy in a given patient. In any event, and regardless of call type, once patient care is engaged, an ambulance unit is a "deployed asset" and unable to respond to another call until returned to service.

\section{CONCLUSIONS}

EMS data rapidly demonstrated signs of local and systemic health care systems stress. Municipal prehospital databases should be recognized as valuable repositories for actionable intelligence for public health preparedness and response and should be supported to achieve essential societal roles. Specific medical and trauma conditions, criticality, and specific hospitals with increased patient loads were easily identified and their compromised function resolved with the recovery of a safety net academic medical center. Loss of this safety net hospital produced a prolonged disproportionate burden on prehospital transports to surrounding hospitals, as acute disaster conditions became chronically manifest until full restoration of the safety net. Calibrated redeployment of human, economic, and social capital across hospital systems is required to ensure rapid regional recovery.

\section{SUPPLEMENTARY MATERIAL}

To view supplementary material for this article, please visit http://dx.doi.org/10.1017/dmp.2015.175

\section{About the Authors}

Ronald O. Perelman Department of Emergency Medicine, NYU School of Medicine, New York, New York (Drs Smith, Portelli, Lee, and Goldfrank and Ms Malik); Office of Medical Affairs, The Fire Department of the City of New York, New York, New York (Mr Braun, Drs Asaeda and Prezant, and Ms Lancet); Department of Population Health, Division of Biostatistics, NYU School of Medicine, New York, New York (Drs Wang and Hu); and Department of Population Health, NYU School of Medicine, New York, New York (Dr Lee).

Correspondence and reprint requests to Silas W. Smith, MD, Ronald O. Perelman Department of Emergency Medicine, Bellevue Hospital Center, 462 First Avenue, Room A-345A, New York, New York 10016 (e-mail: Silas.Smith@nyumc.org).

\section{Acknowledgments}

This work was funded by the US Department of Health and Human Services, Office of the Assistant Secretary for Preparedness and Response, award number HITEP130006-01-00 to the NYU School of Medicine. Additional funding was received for follow-on studies from ASPR, award number HITEP 150030-01-00 to the NYU School of Medicine. SWS derives additional salary support from the Fridolin Charitable Trust to the Ronald O. Perelman Department of Emergency Medicine Safety Program and has received an intramural departmental 2015 scholarly innovation grant for work unrelated to the current study. DJP receives additional support from FDNY for work unrelated to the current study. $\mathrm{MH}$ is supported by an intramural NYU
School of Medicine research grant and grant NIH/NCI 5 P30 CA16087-32 to the NYU School of Medicine for work unrelated to the current study; he has a pending grant NIH/NCI 1R01CA205029-01 for work unrelated to the current study. The funding agency (ASPR) played no role in the design or conduct of the study; collection, management, analysis, or interpretation of the data; preparation of the manuscript; or decision to publish.

\section{Disclaimer}

The content of this article is the responsibility of the authors and does not necessarily represent the official views of the US Department of Health and Human Services, the Office of the Assistant Secretary for Preparedness and Response, the NYU School of Medicine, the NYC Health and Hospital Corporation, Bellevue Hospital Center, The Fire Department of the City of New York, or any employers, affiliations, named entities, or other funding agencies or sources.

Published online: January 7, 2016.

\section{REFERENCES}

1. Institute of Medicine. Committee on Guidance for Establishing Crisis Standards of Care for Use in Disaster Situations. Crisis Standards of Care. A Systems Framework for Catastrophic Disaster Response. Washington, DC: National Academies Press; 2012.

2. Institute of Medicine. Committee on the Future of Emergency Care in the United States Health System. Hospital-Based Emergency Care: At the Breaking Point. Washington, DC: National Academies Press; 2006.

3. US Department of Homeland Security. NIPP 2013: Partnering for Critical Infrastructure Security and Resilience. http://www.dhs.gov/sites/default/ files/publications/National-Infrastructure-Protection-Plan-2013-508.pdf. Published 2013. Accessed August 21, 2015.

4. Lurie N, Manolio T, Patterson AP, et al. Research as a part of public health emergency response. N Engl J Med. 2013;368(13):1251-1255. http://dx.doi.org/10.1056/NEJMsb1209510.

5. Institute of Medicine. Enabling Rapid and Sustainable Public Health Research During Disasters: Summary of a Joint Workshop by the Institute of Medicine and the US Department of Health and Human Services. Washington, DC: The National Academies Press; 2014.

6. United States Government Accountability Office. National Preparedness: Improvements Needed for Measuring Awardee Performance in Meeting Medical and Public Health Preparedness Goals. Report to the Committee on Energy and Commerce, House of Representatives. Report Number: GAO-13-278. Washington, DC: United States Government Accountability Office; 2013.

7. New York Academy of Medicine. Institute of Medicine. Identifying Disaster Medical and Public Health Research Priorities: Data needs arising in response to Hurricane Sandy. Meeting Summary. November 16, 2012. New York, NY: The New York Academy of Medicine; 2012.

8. Lee DC, Smith SW, McStay CM, et al. Rebuilding emergency care after Hurricane Sandy. Disaster Med Public Health Prep. 2014; Apr 9 1-4. [Epub ahead of print].

9. Munjal KG, Silverman RA, Freese J, et al. Utilization of emergency medical services in a large urban area: description of call types and temporal trends. Prehosp Emerg Care. 2011;15(3):371-380. http://dx.doi. org/10.3109/10903127.2011.561403.

10. Freese J, Richmand NJ, Silverman RA, et al. Impact of a citywide blackout on an urban emergency medical services system. Prehosp Disaster Med. 2006;21(06):372-378. http://dx.doi.org/10.1017/S1049023X00004064.

11. Prezant DJ, Clair J, Belyaev S, et al. Effects of the August 2003 blackout on the New York City healthcare delivery system: a lesson for disaster preparedness. Crit Care Med. 2005;33(1)(suppl):S96-S101. http://dx.doi. org/10.1097/01.CCM.0000150956.90030.23.

12. Kue RC, Dyer KS. The impact of heat waves on transport volumes in an urban emergency medical services system: a retrospective review. Prehosp Disaster Med. 2013;28(06):610-615. http://dx.doi.org/10.1017/S1049023X 13008960. 
13. El Sayed M, Mitchell PM, White LF, et al. Impact of an emergency department closure on the local emergency medical services system. Prehosp Emerg Care. 2012;16(2):198-203. http://dx.doi.org/10.3109/10903127. 2011.640418.

14. Auf der Heide E. The importance of evidence-based disaster planning. Ann Emerg Med. 2006;47(1):34-49. http://dx.doi.org/ 10.1016/j.annemergmed.2005.05.009.

15. Smith SW, Portelli I, Narzisi G, et al. A novel approach to multihazard modeling and simulation. Disaster Med Public Health Prep. 2009;3 (02):75-87. http://dx.doi.org/10.1097/DMP.0b013e3181a88899.

16. Adalja AA, Watson M, Wollner S, et al. Response to the sudden closure of St. Vincent's Hospital: learning from a real, no-notice, prolonged surge event. Biosecur Bioterror. 2011;9:153-161.

17. Romero D, Kwan A, Nestler S, et al. Impact of the closure of a large urban medical center: a quantitative assessment (part II). J Community Health. 2012;37(5):995-1005. http://dx.doi.org/10.1007/s10900-0129551-2.

18. Raven MC, Carrier ER, Lee J, et al. Substance use treatment barriers for patients with frequent hospital admissions. J Subst Abuse Treat. 2010; 38(1):22-30. http://dx.doi.org/10.1016/j.jsat.2009.05.009.

19. McCormack RP, Hoffman LF, Wall SP, et al. Resource-limited, collaborative pilot intervention for chronically homeless, alcoholdependent frequent emergency department users. Am J Public Health. 2013;103(S2)(suppl 2):S221-S224. http://dx.doi.org/10.2105/AJPH.2013. 301373.

20. McCormack RP, Hoffman LF, Norman M, et al. Voices of homeless alcoholics who frequent Bellevue Hospital: a qualitative study. Ann Emerg Med. 2015;65:178-86 e6. http://dx.doi.org/10.1016/j.annemergmed. 2014.05.025.
21. Fishman PE, Shofer FS, Robey JL, et al. The impact of trauma activations on the care of emergency department patients with potential acute coronary syndromes. Ann Emerg Med. 2006;48(4):347-353. http:// dx.doi.org/10.1016/j.annemergmed.2006.02.021.

22. Larson DM, Menssen KM, Sharkey SW, et al. "False-positive" cardiac catheterization laboratory activation among patients with suspected STsegment elevation myocardial infarction. JAMA. 2007;298 (23):2754-2760. http://dx.doi.org/10.1001/jama.298.23.2754.

23. Arya R, Dossantos F, Ohman-Strickland P, et al. Impact of trauma activation on the ED length of stay for nontraumatic patients. Am J Emerg Med. 2012;30(2):311-316. http://dx.doi.org/10.1016/j.ajem. 2010.12.011.

24. Guttmann A, Schull MJ, Vermeulen MJ, et al. Association between waiting times and short term mortality and hospital admission after departure from emergency department: population based cohort study from Ontario, Canada. BMJ. 2011;342:d2983. http://dx.doi.org/10.1136/ bmj.d2983.

25. Zigmond J. New Orleans charity care soars. Huge decline in staffed beds adds to regional strain. Mod Healthc. 2006;36:14-15.

26. Rudowitz R, Rowland D, Shartzer A. Health care in New Orleans before and after Hurricane Katrina. Health Aff (Millwood). 2006;25(5): w393-w406. http://dx.doi.org/10.1377/hlthaff.25.w393.

27. Redlener I, Reilly MJ. Lessons from Sandy-preparing health systems for future disasters. N Engl J Med. 2012;367(24):2269-2271. http://dx.doi. org/10.1056/NEJMp1213486.

28. Raven MC, Lowe RA, Maselli J, et al. Comparison of presenting complaint vs discharge diagnosis for identifying "nonemergency" emergency department visits. JAMA. 2013;309(11):1145-1153. http:// dx.doi.org/10.1001/jama.2013.1948. 